\title{
Long-term complete response of antiandrogen withdrawal syndrome in a patient with metastatic prostate cancer: A case report
}

\author{
MASAYUKI SANO, SHINYA YAMAMOTO, SHO UEHARA, TAKESHI YUASA, \\ HITOSHI MASUDA, IWAO FUKUI and JUNJI YONESE
}

Department of Urology, Cancer Institute Hospital, Japanese Foundation for Cancer Research, Tokyo 135-8550, Japan

Received March 16, 2016; Accepted May 26, 2016

DOI: $10.3892 / \mathrm{mco} .2016 .946$

\begin{abstract}
Antiandrogen withdrawal syndrome (AWS) is a well-established phenomenon in prostate cancer treated with combined androgen blockade (CAB). AWS is generally defined as subjective and/or objective improvement following discontinuation of an antiandrogen. However, the duration of the AWS response is usually limited. In addition, a complete response is quite rare. We herein present the case of a patient who achieved complete response from AWS, with the duration of this response lasting for $>6$ years. A 72-year-old man with metastatic prostate cancer received $\mathrm{CAB}$ with a luteinizing hormone-releasing hormone analog and bicalutamide. In addition, for local cancer control, external beam radiation therapy (70 Gy) to the prostate was performed. Subsequently, the serum prostate-specific antigen (PSA) level reached a nadir (undetectable level). Four years later, the patient's serum PSA level started to rise, and bicalutamide was discontinued to confirm AWS at a serum PSA level of $0.34 \mathrm{ng} / \mathrm{ml}$. The PSA level immediately decreased again to an undetectable level $(0.00 \mathrm{ng} / \mathrm{ml})$, where it has been remained for 6 years. Bone scintigraphy and computed tomography scans have shown no evidence of bone or other metastases since the introduction of AWS. To the best of our knowledge, there have been no reports of such a long duration of complete response from AWS. Therefore, this phenomenon should always be considered, even in patients with advanced disease.
\end{abstract}

Correspondence to: Dr Masayuki Sano, Department of Urology, Cancer Institute Hospital, Japanese Foundation for Cancer Research, 3-8-31 Ariake, Koto, Tokyo 135-8550, Japan

E-mail: sano919@nms.ac.jp

Abbreviations: AWS, antiandrogen withdrawal syndrome; PSA, prostate-specific antigen; $\mathrm{CAB}$, combined androgen blockade; CT, computed tomography; AR, androgen receptor

Key words: prostate cancer, antiandrogen withdrawal syndrome, bicalutamide, complete response, bone metastases

\section{Introduction}

Progressive prostate cancer that has been treated with androgen-deprivation therapy using an antiandrogen, such as bicalutamide, occasionally causes antiandrogen withdrawal syndrome (AWS), which results in a response involving a significant decline in prostate-specific antigen (PSA) levels.

AWS is generally defined as subjective and/or objective improvement following discontinuation of an antiandrogen in patients with elevated PSA levels who are treated with combined androgen blockade (CAB) using steroidal or non-steroidal antiandrogens (1).

The duration of AWS varies considerably (1) and only a limited number of cases exhibiting a complete response, in terms of PSA level decline, have been reported.

We herein report the case of a patient with metastatic prostate cancer who received $\mathrm{CAB}$, with the longest duration of a complete response reported to date.

\section{Case report}

A 72-year-old man visited the Cancer Institute Hospital (Tokyo, Japan) on June 9, 2004, complaining of right hip joint pain. Laboratory examination revealed a PSA level of $588.8 \mathrm{ng} / \mathrm{ml}$ and an elevated alkaline phosphatase level 539 IU/1 (normal range, 100-325 IU/1). Prostate biopsy revealed adenocarcinoma, with a Gleason score of $4+3=7$. Computed tomography (CT) and bone scintigraphy scans revealed metastases to the right pelvis and right femoral head (extent of disease I).

$\mathrm{CAB}$ consisting of a luteinizing hormone-releasing hormone analog and bicalutamide $80 \mathrm{mg} /$ day was immediately administered. The serum PSA level promptly decreased to $0.16 \mathrm{ng} / \mathrm{m}$ in 3 months and had reached a nadir of $0.02 \mathrm{ng} / \mathrm{m}$ by May 26, 2005. External beam radiation therapy ( $70 \mathrm{~Gy}$ ) to the prostate was then performed for local cancer control. The PSA level had decreased to $0.00 \mathrm{ng} / \mathrm{ml}$ by January 31, 2006. In addition, no bone metastases were detected on bone scintigraphy.

The PSA level gradually increased to $0.34 \mathrm{ng} / \mathrm{m}$ by March 3, 2009; however, no new lesions of distant metastasis were detected by CT or bone scintigraphy. Bicalutamide was discontinued to confirm AWS. The PSA level again decreased to $0.00 \mathrm{ng} / \mathrm{ml}$ by August 18, 2009. To date, this undetectable 


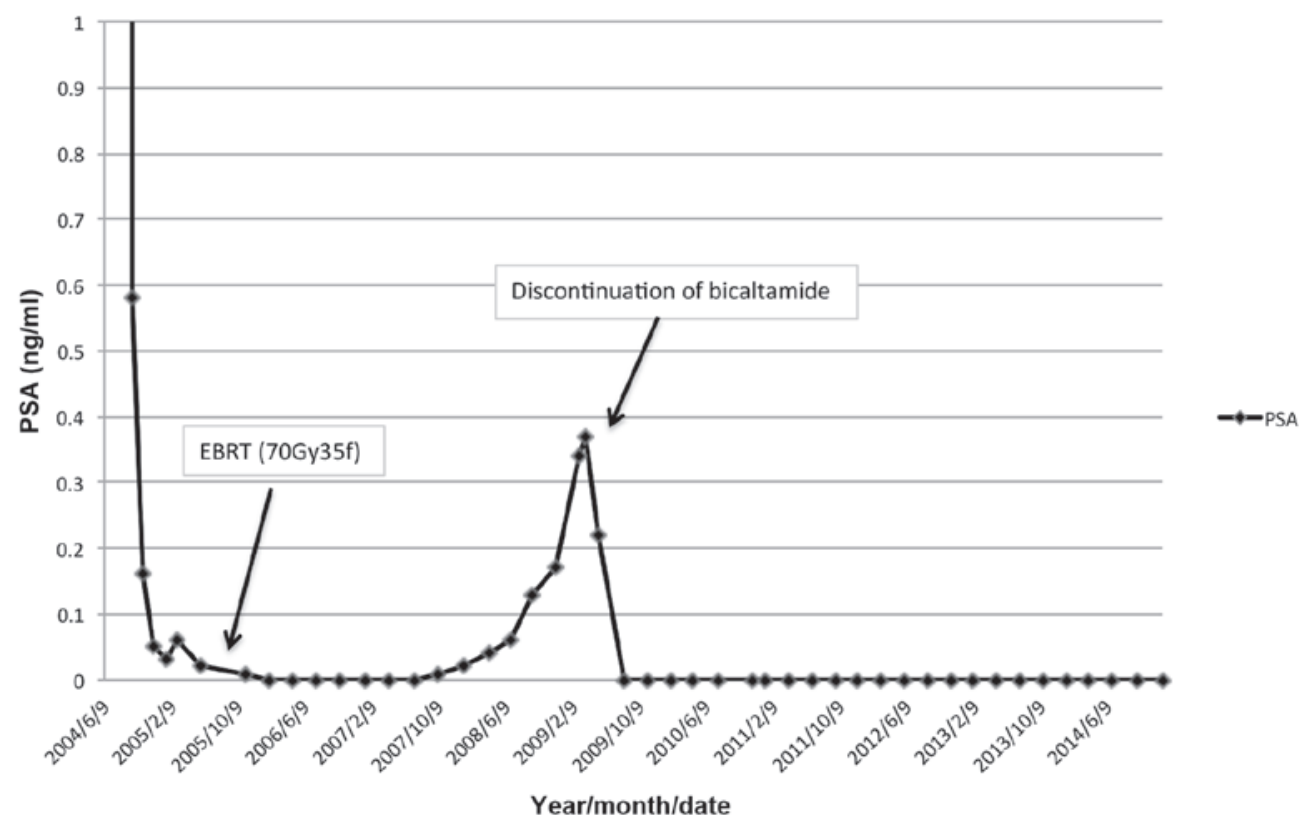

Figure 1. Change in serum PSA concentration in the patient treated with combined androgen blockade and after cessation of the non-steroidal antiandrogen bicalutamide. EBRT, external beam radiation therapy; PSA, prostate-specific antigen.

PSA level has been maintained, and no recurrence has been detected by bone scintigraphy or $\mathrm{CT}$ for the past 6 years since the introduction of AWS. The clinical course and change in serum PSA levels are shown in Fig. 1.

Written informed consent was obtained from the patient regarding the publication of the details of this case.

\section{Discussion}

AWS is a well-established phenomenon in prostate cancer (1). Kelly and Sher first reported AWS with the non-steroidal antiandrogen flutamide (2); they reported a decrease of $\geq 50 \%$ in the PSA level following discontinuation of flutamide in 10 of their 35 patients (29\%) (3).

Small et al first reported AWS with bicalutamide in 1994 (4). The association between antiandrogen withdrawal and a change in PSA level is a general phenomenon observed for all antiandrogens. The differences in the binding affinity of various antiandrogens are unrelated. The response rate previously reported by Suzuki et al was $15.5 \%$ for bicalutamide and $12.8 \%$ for flutamide, with no significant difference between the two antiandrogens (5) and a reported mean response duration of 5.8 \pm 3.8 months. Noguchi et al reported the case of a patient who exhibited a complete PSA decline for $>2$ years after the discontinuation of a steroidal antiandrogen (6). Compared to these previously reported studies, the response duration of the present case is the longest reported to date.

The duration of antiandrogen use prior to withdrawal was identified as an AWS predictor by Sator et al (7), who reported that only the duration of prior antiandrogen exposure was a significant predictor of PSA response after withdrawal. Additionally, a higher frequency of AWS was observed in the group of patients who received antiandrogen for a longer duration ( $>32$ months prior to withdrawal). In the present case, consistent with Sator's report, the patient received bicalutamide for $\sim 48$ months prior to withdrawal.
The molecular mechanisms underlying AWS have not been fully elucidated. Mutation of androgen receptors (ARs) is the most supported hypothesis (8). In this hypothesis, bicalutamide acts as an agonist for mutant ARs. In the present case, a luteinizing hormone-releasing agonist was used for $>4$ years, and external beam radiation $(70 \mathrm{~Gy} / 35 \mathrm{fx})$ was performed prior to the discontinuation of bicalutamide. It is suggested that, during this period, androgen-dependent and hormone-sensitive tumor cells were eliminated, whereas a moderate number of AR-mutated cells survived.

Takeshita et al reported a case in which AWS and radiotherapy of the prostate contributed to the disappearance of metastatic lymph nodes and a decline in PSA level to below the detection limit for a long period (9). Culp et al recently reported that local therapy for the prostate may confer a survival advantage to selected patients with metastatic disease (10). Therefore, in the present case, AWS combined with local treatment to the prostate (radiation therapy) may achieve good cancer control.

The median overall survival following antiandrogen withdrawal was 22 months (7). Small et al reported that there was no significant difference between the median survival times of AWS responders and non-responders (11). However, despite advanced disease, the patient in the present case has survived for $>10$ years since the diagnosis. It is thus considered that AWS has contributed to the prolongation of the patient's life expectancy and has improved his quality of life.

In conclusion, although the long response duration seen in the present case is rare, the AWS phenomenon should be considered, even in patients with advanced disease.

\section{Acknowledgements}

The authors would like to thank Enago (www.enago.jp) for the English language review. 


\section{References}

1. Paul R and Breul J: Antiandrogen withdrawal syndrome associated with prostate cancer therapies: Incidence and clinical significance. Drug Saf 23: 381-390, 2000.

2. Kelly WK and Scher HI: Prostate-specific antigen decline after antiandrogen withdrawal: The flutamide withdrawal syndrome. J Urol 149: 607-609, 1993.

3. Scher HI and Kelly WK: Flutamide withdrawal syndrome: Its impact on clinical trials in hormone-refractory prostate cancer. J Clin Oncol 11: 1566-1572, 1993.

4. Small EJ and Carroll PR: Prostate-specific antigen decline after casodex withdrawal: Evidence for an antiandrogen withdrawa syndrome. Urology 43: 408-410, 1994.

5. Suzuki H, Okihara K, Miyake H, Fujisawa M, Miyoshi S, Matsumoto T, Fujii M, Takihana Y, Usui T, Matsuda T, et al: Alternative nonsteroidal antiandrogen therapy for advanced prostate cancer that relapsed after initial maximum androgen blockade. J Urol 180: 921-927, 2008.

6. Noguchi K, Teranishi J, Uemura H, Fujikawa N, Saito K and Murai T: Complete response, as determined by prostate-specific antigen level, to chlormadinone acetate withdrawal persisting longer than 2 years in patients with advanced prostate cancer: Two case reports. Int J Urol 13: 1259-1261, 2006.
7. Sartor AO, Tangen CM, Hussain MH, Eisenberger MA, Parab M, Fontana JA, Chapman RA, Mills GM, Raghavan D and Crawford ED; Southwest Oncology Group: Antiandrogen withdrawal in castrate-refractory prostate cancer: A Southwest oncology group trial (SWOG 9426). Cancer 112: 2393-2400, 2008.

8. Hara T, Miyazaki J, Araki H, Yamaoka M, Kanzaki N, Kusaka M and Miyamoto M: Novel mutations of androgen receptor: A possible mechanism of bicalutamide withdrawal syndrome. Cancer Res 63: 149-153, 2003.

9. Takeshita H, Kawakami S and Fukui I: Profound bicalutamide withdrawal syndrome in a hormone-refractory T4N1 prostate cancer permitting both salvage radiotherapy and cessation of hormonal therapy. Int J Urol 16: 337-338, 2009.

10. Culp SH, Schelhammer PF and Williams MB: Might men diagnosed with metastatic prostate cancer benefit from definitive treatment of the primary tumor? A SEED-based study. Eur Urol 65: 1058-1066, 2014

11. Small EJ and Srinivas S: The antiandrogen withdrawal syndrome. Experience in a large cohort of unselected patience with advanced prostate cancer. Cancer 76: 1428-1434, 1995. 